\title{
Utilizing Quantitative Phase Microscopy to Observe Cellular Response to Treatment and Dynamic Behaviors
}

\author{
Katherine Creath ${ }^{1,2,3}$ and Goldie Goldstein ${ }^{1}$ \\ 1. 4D Technology Corp., Tucson, AZ, USA \\ 2. College of Optical Sciences, University of Arizona, Tucson, AZ, USA \\ 3. Optineering, Tucson, AZ, USA
}

The ability to view cells' direct response before, during and after exposure to a treatment or drug is an important means to discovering reaction mechanisms and morphology changes. One new method that is providing new insight into dynamic cellular morophology is quantitative phase microscopy. This labelfree imaging modality shows great utility in its ability to examine optical thickness and volume changes, directly proportional to dry cell mass over a range of timescales [1]. This paper describes new and recent research related to measuring optical thickness via a quantitative phase microscope and using it to study small populations of beating cardiac myocytes' and their response to a drug. Additional measurements are shown of blood flow in a 3-day old zebrafish and vesicle motion within actin fibers in myoblasts.

With quantitative phase microscopy, it is possible to quantify optical thickness values (actual integrated physical thickness times index of refraction) at fast time scales not possible by other techniques. Areas that have higher index of refraction are more optically dense and appear thicker $[2,3]$. The microscope developed for this research utilizes a Linnik interference microscope with epi-illumination as described in Biomedical Optics Express [3]. It operates at a wavelength of $511 \mathrm{~nm}$ or $660 \mathrm{~nm}$ and utilizes a Linnik interference objective at a magnification of 20X NA 0.45 and 50X NA 0.8 with additional magnification obtained via a flip-in 2X field of view (FOV) lens. The camera is an Illunis XMV-2020 with a Moxtek pixelated phase mask to enable spatial phase measurement with short exposures $(<1 \mathrm{~ms})$ at frame rates as high as $15 \mathrm{fps}$ to freeze motion while being insensitive to vibration [3,4]. For the examples in this paper, objects are viewed in double pass on a reflective surface as shown in Figure 1.

Quantitative phase information is extremely sensitive to observe cells' response to a treatment or drug. Cardiac myocytes from 1-2 day old neonatal Sprague Dawley (Harlan, Indianapolis, IN) rats were prepared at the University of Arizona and plated onto \#1 round coverslips. After 2 weeks of incubation the cells were measured in a Bioptechs FCS3 perfusion chamber with an aluminized base plate and a $100 \mu \mathrm{m}$ gasket. Cells were kept at $37^{\circ} \mathrm{C}$ with an HBSS (Hank's balanced salt solution) fluid bath. Figure 2 shows optical thickness (OT) maps of an area of cells imaged at 40X (20X with 2X FOV lens) before and after pushing IPHC (isoproterenol hydrochloride - a beta adrenergic agonist). These images are the first in two time series of 200 datasets taken at $15 \mathrm{fps}$ over a $13.3 \mathrm{sec}$ time period. These images are the same 349 x 326 pixel subarea of 1050×1200 images. The optical volume of the subareas is determined by summing all the OT values and then scaling so that both traces have the same mean and same relative scaling. The actual physical volume is not obtainable from these data because the thickness of the cell culture and the index of refraction data are unknown.

A major advantage of this technique is that relative physical changes can easily be measured. Figure 2 shows the relative optical volume for these two time series before and after pushing IPHC. These cells 
spontaneously beat about once every four seconds. After the IPHC is pushed, the beating frequency increases by a factor of eight and the flexion is about three times stronger. The optical volume is getting smaller in this subarea when the cells beat because the cells are noticeably stretching out and expanding during the beats.

Dynamic measurements in live organisms can be measured in real-time as shown in Figure 3 showing blood flow through a cardinal vein in a 3-day old zebrafish.

Quantification of local optical volume enables the tracking

of vesicle motion within actin fibers in myoblasts. The optical volume of two clusters can be calculated as the clusters join together. Using conventional techniques, once two particles overlap, they can no longer be distinguished from each other. By utilizing quantitative phase, the optical volume of the vesicles allows for location tracking, even when particles overlap. Myoblasts were measured every minute over the course of 15 minutes. Two clusters were identified to track that cluster together during the course of the measurement, as seen in Figure 4.

These measurements show how quantitative optical phase and optical volume measurements can be used to study a group of cells over time to better understand their function and response to drugs or other treatment. Measurement methods will be described and data movies for these and other examples will be presented.

\section{References:}

[1] R. Barer, "Interference microscopy and mass determination," Nature, 169, pp. 366-367, 1952.

[2] K. Creath, "Dynamic quantitative phase images of pond life, insect wings, and in vitro cell cultures," Proc. SPIE, vol. 7782, pp. 77820B.01-13, 2010.

[3] K. Creath and G. Goldstein, "Dynamic quantitative phase imaging for biological objects using a pixelated phase mask," Biomedical Optics Express, vol. 3, pp. 2866-2880, 2012.

[4] B. T. Kimbrough, "Pixelated mask spatial carrier phase shifting interferometry algorithms and associated errors," Applied Optics, vol. 45, pp. 4554-4562, 2006. [5] The authors acknowledge Charles Crandall, Craig Weber, Ron Lynch, and Jordan Lancaster for their assistance in this project. This work partially supported by NIH/NCRR 1R43RR028170-01, 2R44RR028170-02, and NIH/NIGMS 8R44GM103406-03.

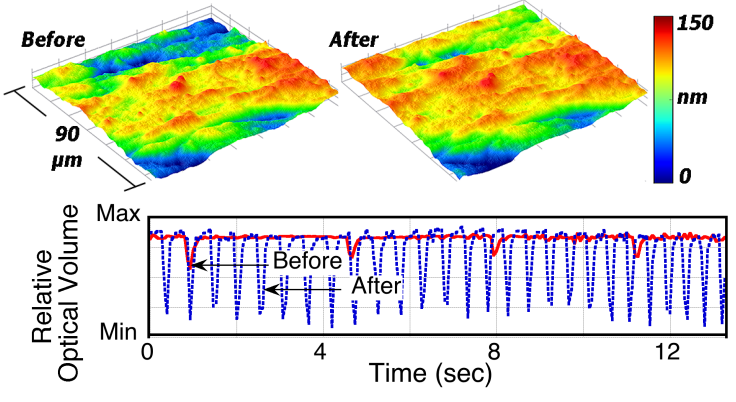

Figure 1: Rat cardiomyocyte OT maps (top left) before and (top right) after pushing IPHC. (bottom) Relative optical volume over time series of 200 datasets.

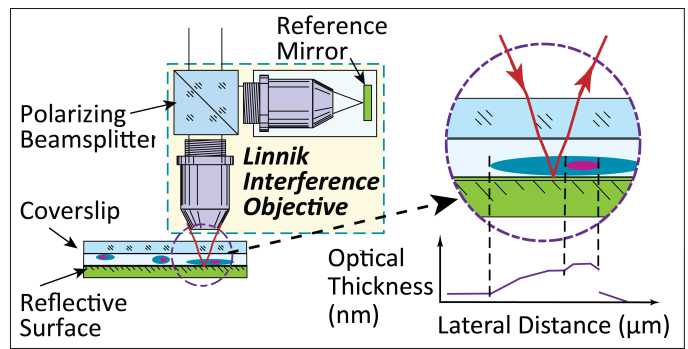

Figure 2. Sample configuration for measurements. Liquid gap is $\sim 100 \mu \mathrm{m}$ with $\# 1$ coverslips $(\sim 150 \mu \mathrm{m})$.

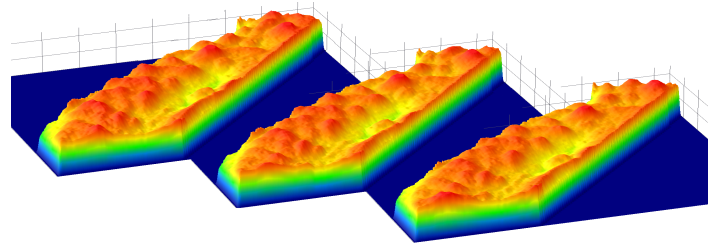

Figure 3. Blood flow in a live 3-day old zebrafish tail.

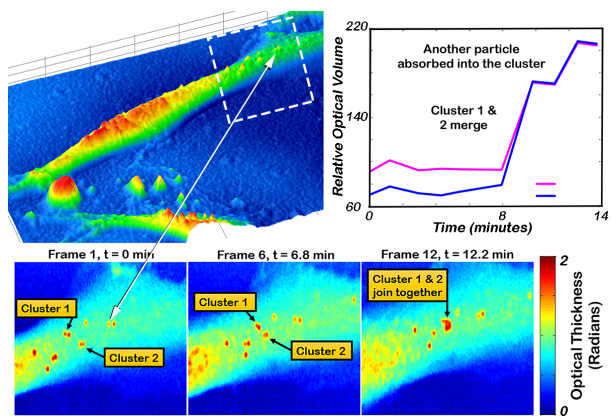

Figure 4: (top left) 3D optical thickness map of a myoblast. (bottom) Zoomed in time series of vesicle flow in highlighted area of myoblast (45 um FOV). (top right) Relative optical volume of vesicle clusters traveling along actin fibers. Tracking of vesicles is possible even when overlap of clusters occurs. 